\title{
Actualización: Vaginismo
}

\author{
Vaginismus
}

Catalina Brosens ${ }^{\star}$, Sergio Terrasa** y Elsa Astolfi***

\begin{abstract}
Resumen
Los autores describen un caso de vaginismo, así como el abordaje empleado por el equipo tratante y la evolución de la paciente. También se explayan sobre las principales características de esta entidad clínica y sobre las formas de trabajo recomendadas por los expertos en el área.
\end{abstract}

\section{Abstract}

The authors describe a case of vaginismus, the used approach by the health team and the evolution of the patient. They also discuss about the principal features of this clinical condition and the management strategies recommended by experts.

Palabras clave: vaginismo, tratamiento. Key words: vaginismus, treatment.

Brosens C, Terrasa S y Astolfi E. Vaginismo. Evid Act Pract Ambul. 12(3): 102-103 Jul-Sep.2009.

\section{Caso clínico}

Una mujer de 22 años oriunda de Formosa concurre a la consulta con su médico de cabecera (que tambien atiende a sus tres hermanas mayores) con quien se conocen desde 2000. Ella está casada hace un año y en pareja estable con su actual marido, hace tres. Durante los últimos meses había presentado cefalea tensional y durante las últimas consultas, se había mostrado con el ánimo triste, con trastornos del sueño y con hipobulia (10/2002). Luego de una primera evaluación, el médico define el diagnóstico de depresión unipolar y la medica con antidepresivos (50mg de sertralina) más clonazepan 0,5mg dos veces por día. Unos meses después (04/2003) la paciente había evolucionado en forma favorable de su síndrome depresivo y acuerda con el médico suspender lentamente el clonazepan y completar seis meses de tratamiento con antidepresivos. Tres meses después (07/2003) la paciente decide consultar por un problema de larga data, pero que hasta el momento no se había atrevido a contar. Avergonzada, cuenta que nunca había podido mantener relaciones sexuales con su pareja, que cada vez que lo intentaban ella sentía tanto dolor ante el mínimo intento de penetración, y que hasta el momento no lo habían logrado. Ella pensaba que las cosas cambiarían con el casamiento, pero luego de un año de casados, y queriendo ahora buscar un embarazo, se animó a consultar. El médico la interroga a solas y la cita con su marido. En la consulta siguiente ambos cónyuges aseguran que más allá de la disfunción sexual están muy bien como pareja y acuerdan realizar una interconsulta con sexología.

\section{Introducción}

El vaginismo es una disfunción sexual femenina que consiste en la contracción involuntaria e inconsciente de los músculos del tercio inferior de la vagina'. Se lo clasifica en distintos grados de acuerdo a la intensidad de la contracción. Se lo denomina severo cuando la contracción es tan intensa que impide la entrada a la vagina; moderado, cuando la intensidad de la contracción es menor y permite la penetración en forma parcial; y leve cuando la penetración es total, pero siempre con dolor ${ }^{2}$. Tambien se clasifica al vaginismo como primario o secundario. Es primario cuando se presenta desde el primer intento de penetración y secundario cuando aparece luego de un período de funcionamiento normal. El vaginismo es una de las causas más frecuentes de que las parejas o matrimonios se consideren "no consumados".

\section{Epidemiología}

El vaginismo tendría una prevalencia cercana al $1 \%$ en la población general ${ }^{3}$. En el consultorio de Sexología del Servicio de Ginecología del Hospital Italiano de Buenos Aires, las consultas por dispareunia, vaginismo y matrimonio no consumado por vaginismo representan aproximadamente el $20 \%$ de la consultas sexológicas femeninas, cuyas edades oscilan entre los 17 y los 66 años (promedio 32). Respecto de 107 consultas por "matrimonio no consumado" el promedio de edad de las pacientes al momento de la consulta fue de 29 años (rango 17 a 52) y habían esperado meses a años hasta consultar.

\section{Fisiopatogenia}

Las causas de vaginismo son, en su gran mayoría, psicológicas. Las más frecuentes son el miedo al dolor, el miedo al embarazo, las experiencias sexuales traumáticas, el temor a contraer una enfermedad de transmisión sexual (ETS) o bien el intenso sentimiento de culpa por haber tenido una educación muy estricta o muy religiosa, en la cual el sexo fue mostrado como algo malo o inexistente y sin que se discutiera sobre el mismo ${ }^{4}$. La contracción de los músculos vaginales funcionaría como un mecanismo de defensa inconsciente para evitar el peligro que implica la penetración.

\section{Diagnóstico}

Es importante descartar otras causas de dispareunia a través del examen físico (alteraciones anatómicas, causas infecciosas, vaginitis atrófica, lubricación inadecuada, etc.). Durante el mismo, la paciente suele estar tensa y con la musculatura perineal y de los muslos, contraída ${ }^{5}$ (p. ej. puede ser dificultoso tomarle la prueba de Papanicolau).

Aclaramos que las mujeres que sufren vaginismo tienen lubricación normal, conservan su deseo sexual y son capaces de tener orgasmos. El vaginismo se asocia a angustia, baja autoestima, miedos y depresión. Es frecuente que las pacientes piensen que son las únicas a quienes les sucede esto, sintiéndose avergonzadas o anormales, por lo que tardan mucho en consultar.

Con frecuencia, deciden hacerlo cuando desean un embarazo o cuando la desesperación o el miedo al abandono superan a la vergüenza. El vaginismo puede ser no diagnosticado o mal diagnosticado confundiéndolo con un anillo fibroso o un himen rígido, lo que puede conducir a una cirugía innecesaria e inútil.

También es frecuente que a estas pacientes se les realice el examen ginecológico vaginal de manera forzada, lo que tiende a acentuar los síntomas. Vale aclarar que una de las principales dificultades para el diagnóstico de esta entidad es la deficiente preparación de los profesionales de la salud en el área de la salud sexual.

* Servicio de Medicina Familiar y Comunitaria del Hospital Italiano de Buenos Aires.

** Servicio de Medicina Familiar y Comunitaria del Hospital Italiano de Buenos Aires.

*** Servicio de Ginecología del Hospital Italiano de Buenos Aires. 


\section{Tratamiento}

La mayoría de los tratamientos están enmarcados en el paradigma cognitivo-conductual y tienen por objetivo ayudar a la mujer a ganar el control sobre sus músculos del piso de la pelvis ${ }^{1}$, se recomienda un abordaje interdisciplinario que sean interdisciplinarios. Algunos expertos recomiendan que el tratamiento incluya algún abordaje psicoterapéutico para la baja autoestima, la angustia, el miedo y la eventual depresión, que pueden estar asociados a esta condición clínica. Por ejemplo, puede ser muy terapéutico intentar que la mujer ponga en palabras todos sus miedos y fantasías. Por un lado, se exploran los temores relacionados con el coito y se trabaja sobre ellos. También es útil explicar a la paciente la anatomía de la vagina y el funcionamiento de los músculos que están provocando el síntoma: que éstos están respondiendo a una orden de defensa inconsciente que ella no está pudiendo modificar voluntariamente. En algunas pacientes es necesaria la medicación de los trastornos de la ansiedad o de alguna otra co-morbilidad psiquiátrica.

En cuanto a lo específicamente dirigido al vaginismo, el tratamiento incluye ejercicios de exploración de la vagina a través del tacto y la desensibilización progresiva con el uso de dilatadores vaginales. Se le dice a la paciente que, con la ayuda de lubricantes, inserte en su vagina objetos de forma fálica que van aumentando gradualmente de tamaño. Por otro lado, se le recomienda la realización de ejercicios de Kegel para la contracción y relajación voluntaria y consciente de los músculos perineales (también utilizados en el tratamiento de la incontinencia). Con estos últimos se intenta que la mujer tenga mayor habilidad para identificar los músculos implicados en lo que le está ocurriendo. Por ejemplo, la mujer coloca un dedo en su vagina intentando "apretar" el dedo y luego relajarlo utilizando sólo los músculos vaginales y evitando utilizar los glúteos, los muslos y los abdominales ${ }^{1}$. Se recomienda que las sesiones con el euipo de salud tengan una frecuencia semanal. Todos estos ejercicios se realizan con el objeto de enseñar a la paciente que el control de la musculatura vaginal puede ser voluntario e indoloro, tratándose de un proceso en el que la mujer toma un rol activo. Por esto es muy importante evitar provocar dolor durante el examen físico, y decirle a la paciente que es ella quien tiene el control. Se debe transmitirle a la paciente que mientras dure la terapia la pareja no deberá intentar la penetración.

Sobre estas consignas básicas se pueden aplicar muchas variantes con el fin de adecuar la terapia a cada paciente (p. ej. si tiene pareja, si la misma colabora o no). Es importante también tener en cuenta la reacción de la pareja frente a este problema, lo que puede influir en la evolución de la terapia. Generalmente dispuestos a colaborar, están los que son comprensivos y tolerantes, los que se sienten culpables, los que lo toman como un rechazo personal, lo que están dolidos o enojados, etc.

La duración de la terapia sexual depende de la velocidad a la que se realiza la desensibilización, oscilando en términos generales desde unas pocas sesiones hasta alrededor de un año. En este sentido, la terapia sexual se considera exitosa y finaliza cuando la mujer puede tener penetración vaginal durante la relación sexual, sin dolor ni molestias. Hay que advertirle, sin embargo, que es muy posible que la penetración no le resulte placentera las primeras veces; y que el placer irá apareciendo gradualmente con el tiempo y la confianza.

De acuerdo a lo reportado por Master y Johnsons ${ }^{6}$, la tasa reportada de éxito de las técnicas de desensibilización con dilatadores vaginales es cercana al $100 \%$ en pacientes/parejas con buena adherencia al tratamiento. Sin embargo, esta evidencia proviene básicamente de estudios no controlados, como lo documentó la revisión sistemática publicada en 2003 por McGuire y col. acerca de la eficacia de los diferentes tratamientos para el vaginismo. Entre otros abordajes posibles y avalados por el momento por estudios no controlados ${ }^{1}$, mencionamos también a las inyecciones con toxina botulínica para los casos refractarios a las estrategias previamente descriptas.

\section{Resolución del caso}

La paciente comenzó a hacer tratamiento con la Médica Sexóloga la semana siguiente luego de que realizara la consulta de pareja (08/2003). Aunque los resultados fueron lentos, el tratamiento fue exitoso y la pareja logró "consumar" su matrimonio a finales de 2004. Unos meses después volvieron a tener algunas dificultades que se resolvieron al retomar el tratamiento. La paciente consultó a ginecología por primera vez (04/2005) y quedó embarazada (06/2005). Durante 2006 y 2007 continuó con el tratamiento sexológico, embarazándose nuevamente (09/2008). A lo largo de estos años, los síntomas reaparecieron esporádicamente, continuando la paciente con el tratamiento. Sin embargo, su satisfacción con el tratamiento y sus resultados es buena.

\section{Conclusión}

Vale aclarar que la duración del tratamiento del vaginismo no suele ser tan prolongada como la del de la paciente presentada como ejemplo. Por último, podemos mencionar que los médicos de atención primaria se encuentran en una posición valiosa para ocuparse de las preocupaciones sexuales de sus pacientes. Esto se debe al seguimiento longitudinal de los pacientes, a su abordaje biopsicosicial, a la confianza que depositan los pacientes en el equipo de salud, y a que muchas veces el médico atiende a los dos miembros de la pareja. Por otro lado, se sabe que la mayoría de los pacientes espera que sea el médico quien pregunte acerca de aspectos vinculados a la vida sexual, y que solo pocos médicos interrogan sobre estos temas ${ }^{8}$. Esto generalmente se debe a la incomodidad de los médicos de hablar sobre estos temas y al miedo de no poder contener o manejar los problemas de la esfera sexual una vez planteados ${ }^{9}$. Por todo esto, consideramos que sería importante que los miembros del equipo de salud pudieran abordar las barreras personales y/o profesionales que los frenan para hablar con sus pacientes acerca de las preocupaciones sexuales de estos, y de esta forma poder ofrecerles una atención más integral.

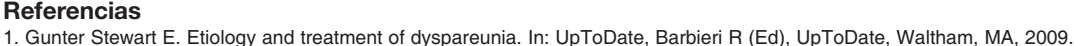

1. Gunter Stewart E. Etiology and treatment of dyspareunia. In: UpToDate, Barbieri R (Ed), UpToDate,
2. Astolfi, E. Disfunciones sexuales femeninas: ¿Qué es el vaginismo?; Aprender Salud; HIBA; 2007.

3. Ferreira A y col. Prevalência das disfunções sexuais femininas em clínica de planejamento familiar de um hospital escola no Recife, Pernambuco. Rev. Bras. Saude Mater. Infant. 2007 Apr ; 7(2): 143-150. Available from: http://www.scielo.br/scielo.php?script=sci_arttext\&pid=S1519-38292007000200004\&Ing=en. doi: 10.1590/S1519-38292007000200004.

4. Reissing E y col. Does vaginismus exist? A critical review of the literature; The Journal of Nervous and Mental Disease; 1999; 187 (5): 261-271.

5. Caplan $\mathrm{H}$ y col.. An Effective Clinical Approach to Vaginismus-Putting the Patient in Charge; The Western Journal of Medicine; Dec. 1988; 149, 6; 769-770.

6. Citado por: Singer Kaplan H. La nueva terapia sexual. 1ra ed.-Buenos Aires: Alianza, 1996. Capítulo 20. Pagina 563.

7. McGuire H y col. Interventions for vaginismus. Cochrane Database of Systematic Reviews. 2003; (1): CD001760.

8. Holzapfel S. Sexual Medicine in Family Practice. Can Fam Physician 1993; 39:608-624.

9. Merryg Gott y col, "Opening a can o worms": GP and practice nurse barriers to talking about sexual health in primary care, Family Practice 2004; $21: 528-536$. 